\title{
Hamiltonian for a string in a Newton-Cartan background
}

\author{
Josef Klusoň* \\ Department of Theoretical Physics and Astrophysics, Faculty of Science, Masaryk University, \\ Kotlářská 2, 61137 Brno, Czech Republic
}

(Received 4 May 2018; published 10 October 2018)

\begin{abstract}
This paper is devoted to the construction of the Hamiltonian for nonrelativistic string in the Newton-Cartan background. We start with the Hamiltonian for relativistic string in general background. Then we perform a limiting procedure on the metric that leads to Newton-Cartan background. We determine the constraint structure for nonrelativistic string and show that these constraints are the first class constraints. Then we determine the corresponding Lagrangian and discuss its properties.
\end{abstract}

DOI: $10.1103 /$ PhysRevD.98.086010

\section{INTRODUCTION}

Recently, non-Lorentzian geometry has gained interest in the theoretical physics community for many reasons. First, today it is well known that strong correlated systems in condensed matter can be successfully described with the help of non-relativistic holography [1-3], for a review see, e.g., [4]. This duality is based on the idea that the strongly coupled theory on the boundary can be described by string theory in the bulk. Further, when the curvature of the spacetime is small we can use the classical gravity instead of the full string theory machinery. In the case of nonrelativistic holography the situation is even more interesting since we have basically two possibilities: Either we use Einstein metric with nonrelativistic isometries [5-7] or we introduce nonrelativistic gravities in the bulk [8,9], like NewtonCartan gravity [10] ${ }^{1}$ or Hořava gravity [25]. It is also very instructive to analyze extended objects in Newton-Cartan theory $[26,27] .{ }^{2}$ In $[26]$ the action for nonrelativistic string in Newton-Cartan background was proposed, which has many interesting properties. For example, in was argued in [26] that in order to define correctly an action for a nonrelativistic string in Newton-Cartan background two longitudinal directions have to be selected and hence we obtain a more general form of the Newton-Cartan geometry. The canonical analysis of this string was performed recently in [30]. During this analysis we met an obstacle which was an impossibility to derive Hamiltonian constraint for the string

\footnotetext{
*klu@physics.muni.cz

${ }^{1}$ For some recent works, see [11-24].

${ }^{2}$ For the analysis of point particles in this background, see [28,29].

Published by the American Physical Society under the terms of the Creative Commons Attribution 4.0 International license. Further distribution of this work must maintain attribution to the author(s) and the published article's title, journal citation, and DOI. Funded by SCOAP .
}

with nonzero gauge field $m_{\mu}{ }^{a}$ that will be defined in the next section. For that reason we were forced to restrict to the case of zero gauge field $m_{\mu}{ }^{a}$ and then we were able to determine the canonical structure of the nonrelativistic string in Newton-Cartan background. In the same way we proceeded with the case of nonrelativistic p-brane. We defined it using the limiting procedure introduced in [15]. We again found a corresponding action for the nonrelativistic p-brane in Newton-Cartan background and determined the canonical structure for this theory on the condition that the gauge field $m_{\mu}{ }^{a}$ is zero.

The fact that in our previous work we considered the situation when the gauge field $m_{\mu}{ }^{a}$ vanishes is rather unsatisfactory since this field is crucial for the invariance of the theory under Milne boost. It would be nice to develop a full canonical formalism where this field is nonzero. We suggested in the conclusion of our previous paper [30] that one way to proceed is to start with the Hamiltonian for the string in general background and then perform the limiting procedure when we generalize the approach introduced in [15] to the case of two longitudinal directions. Exactly this is the goal of our paper. We start with the Hamiltonian for a relativistic string in a general background, introduce relativistic vierbeins and NSNS two form that are functions of fields that define the Newton-Cartan background. These fields also depend on the free parameter that goes to infinity when we define Newton-Cartan gravity [15]. As a result we will be able to find a corresponding Hamiltonian for the string in Newton-Cartan background. However this is certainly not the end of the story since we have to perform consistency checks of this proposal. Explicitly, we have to show that constraints that define this theory are the first class constraints. It turns out that this is a nontrivial task due to the complicated form of the Hamiltonian. Second, we would like to find the Lagrangian for this nonrelativistic string and investigate how it is related to the Lagrangian density proposed in [26]. To do this we carefully examine 
an invariance of the Hamiltonian constraint under generalized Milne boost. We show that the Hamiltonian constraint can be rewritten with the help of variables that are manifestly invariant under Milne transformation so that the Hamiltonian is invariant too. Then we can proceed to the analysis of the corresponding Lagrangian. As a warm up we consider the case of the nonrelativistic string in flat background. We show that there is a crucial difference between inverse Legendre transformation in case of the relativistic string and nonrelativistic one. Explicitly, we show that in case of nonrelativistic string the Lagrange multipliers corresponding to Hamiltonian and spatial diffeomorphism constraints are determined by projections of the equations of motion for $x^{\mu}$ to longitudinal directions instead of their equations of motion. Then we will be able to find a Lagrangian that agrees with the Lagrangian found in [26]. Further we proceed to the most general case of the nonrelativistic string in Newton-Cartan background where the analysis is much more complicated. Despite this fact we find Lagrangian form of the nonrelativistic string in Newton-Cartan background which is manifestly diffeomorphism invariant and which agrees with the Lagrangian density proposed in [26].

This paper is organized as follows. In the next section (II) we introduce canonical form of the relativistic string action and perform limiting procedure that leads to the Hamiltonian for nonrelativistic string in Newton-Cartan background and determine Poisson algebra of constraints. In Sec. III we find the Lagrangian for nonrelativistic string in Newton-Cartan background. Finally in the conclusion (IV) we outline our results and suggest possible extension of this work.

\section{CANONICAL FORMULATION OF NONRELATIVISTIC STRING IN NEWTON-CARTAN BACKGROUND}

We start with the Nambu-Gotto form of the action for relativistic string in general background

$$
\begin{aligned}
S= & -\tilde{\tau}_{F} \int d \tau d \sigma \sqrt{-\operatorname{det}\left(E_{\mu}{ }^{A} E_{\nu}{ }^{B} \eta_{A B} \partial_{\alpha} x^{\mu} \partial_{\beta} x^{\nu}\right)} \\
& +\tilde{\tau}_{F} \int d \tau d \sigma B_{\mu \nu} \partial_{\tau} x^{\mu} \partial_{\sigma} x^{\nu},
\end{aligned}
$$

where $E_{\mu}{ }^{A}$ is $d$-dimensional vierbein so that the metric components have the form

$$
G_{\mu \nu}=E_{\mu}{ }^{A} E_{\nu}{ }^{B} \eta_{A B}, \quad \eta_{A B}=\operatorname{diag}(-1, \ldots, 1) .
$$

Note that the metric inverse $G^{\mu \nu}$ is defined with the help of the inverse vierbein $E^{\mu}{ }_{B}$ that obeys the relation

$$
E_{\mu}{ }^{A} E^{\mu}{ }_{B}=\delta_{B}^{A}, \quad E_{\mu}{ }^{A} E^{\nu}{ }_{A}=\delta_{\nu}^{\mu} .
$$

Further, $B_{\mu \nu}$ is antisymmetric two form field. Finally $x^{\mu}$, $\mu=0, \ldots, d-1$ are embedding coordinates of the string where the two dimensional world sheet is parametrized by $\sigma^{\alpha} \equiv(\tau, \sigma)$ and $\tilde{\tau}_{F}$ is the string tension that could be eventually rescaled when we define nonrelativistic string.

Our goal is to find Hamiltonian nonrelativistic string in Newton-Cartan background with the help of the following procedure. As the first step we determine Hamiltonian from the action (1). Explicitly, from (1) we find following conjugate momenta

$p_{\mu}=-\tilde{\tau}_{F} E_{\mu}{ }^{A} E_{\nu}{ }^{B} \eta_{A B} \partial_{\beta} x^{\nu} g^{\beta \tau} \sqrt{-\operatorname{det} g_{\alpha \beta}}+\tilde{\tau}_{F} B_{\mu \nu} \partial_{\sigma} x^{\nu}$,

where

$$
g_{\alpha \beta} \equiv G_{\mu \nu} \partial_{\alpha} x^{\mu} \partial_{\beta} x^{\nu}, \quad g^{\alpha \beta} g_{\beta \gamma}=\delta_{\gamma}^{\alpha} .
$$

Using (4) we immediately find that the bare Hamiltonian $H_{B}=\int d \sigma\left(p_{\mu} \partial_{\tau} x^{\mu}-\mathcal{L}\right)$ is zero while we have following two primary constraints

$$
\begin{aligned}
\mathcal{H}_{\tau} \equiv & \left(p_{\mu}-\tilde{\tau}_{F} B_{\mu \rho} \partial_{\sigma} x^{\rho}\right) E^{\mu}{ }_{A} E^{\nu}{ }_{B} \eta^{A B}\left(p_{\nu}-\tilde{\tau}_{F} B_{\nu \sigma} \partial_{\sigma} x^{\sigma}\right) \\
& +\tilde{\tau}_{F}^{2} \partial_{\sigma} x^{\mu} E_{\mu}{ }^{A} \eta_{A B} E_{\nu}{ }^{B} \partial_{\sigma} x^{\nu} \approx 0, \\
\mathcal{H}_{\sigma} \equiv & p_{\mu} \partial_{\sigma} x^{\mu} \approx 0 .
\end{aligned}
$$

Now we are ready to find Hamiltonian for the string in Newton-Cartan background with the help of the nonrelativistic limit of relativistic vierbein $E_{\mu}{ }^{A}$ [15]. However as we argued in our recent paper [30] in order to find correct nonrelativistic limit we have to introduce the generalization of Newton-Cartan gravity following [26]. Explicitly, we split target-space indices $A$ into $A=\left(a^{\prime}, a\right)$ where now $a=0,1$ and $a^{\prime}=2, \ldots, d-1$. Then we introduce $\tau_{\mu}{ }^{a}$ so that we write

$$
\tau_{\mu \nu}=\tau_{\mu}{ }^{a} \tau_{\nu}{ }^{b} \eta_{a b}, \quad a, b=0,1 .
$$

In the same way we introduce vierbein $e_{\mu}{ }^{a^{\prime}}, a^{\prime}=2, \ldots, d-1$ and also introduce gauge field $m_{\mu}{ }^{a}$. The $\tau_{\mu}{ }^{a}$ can be interpreted as the gauge fields of the longitudinal translations while $e_{\mu} a^{\prime}$ as the gauge fields of the transverse translations [26]. Then we can also introduce their inverses with respect to their longitudinal and transverse translations

$$
\begin{aligned}
e_{\mu}{ }^{a^{\prime}} e^{\mu}{ }_{b^{\prime}} & =\delta_{b^{\prime}}^{a^{\prime}}, & e_{\mu}{ }^{a^{\prime}} e^{\nu}{ }_{a^{\prime}}=\delta_{\mu}^{\nu}-\tau_{\mu}{ }^{a} \tau^{\nu}{ }_{a}, \\
\tau^{\mu}{ }_{a} \tau_{\mu}{ }^{b} & =\delta_{a}^{b}, & \tau^{\mu}{ }_{a} e_{\mu}{ }^{a^{\prime}}=0, \quad \tau_{\mu}{ }^{a} e^{\mu}{ }_{a^{\prime}}=0 .
\end{aligned}
$$

Now we are ready to introduce following parametrization of the vierbein ${E_{\mu}}^{A}[15]$

$$
E_{\mu}{ }^{a}=\omega \tau_{\mu}{ }^{a}+\frac{1}{2 \omega} m_{\mu}{ }^{a}, \quad E_{\mu}^{a^{\prime}}=e_{\mu}^{a^{\prime}}
$$


where $\omega$ is a free parameter that we take to infinity when we define the nonrelativistic limit. Note that the inverse vierbein to (9) has the form (up to terms of order $\omega^{-3}$ )

$$
\begin{aligned}
E^{\mu}{ }_{a} & =\frac{1}{\omega} \tau^{\mu}{ }_{a}-\frac{1}{2 \omega^{3}} \tau_{b}^{\mu} m_{\rho}{ }^{b} \tau_{a}^{\rho}, \\
E^{\mu}{ }_{a^{\prime}} & =e^{\mu}{ }_{a^{\prime}}-\frac{1}{2 \omega^{2}} \tau^{\mu}{ }_{a} m_{\rho}{ }^{a} e^{\rho}{ }_{a^{\prime}} .
\end{aligned}
$$

Then with the help of (9) and (10) we obtain following form of the metric $G_{\mu \nu}$ and its inverse

$$
\begin{aligned}
G_{\mu \nu}= & E_{\mu}{ }^{a} E_{\nu}{ }^{b} \eta_{a b}+E_{\mu}{ }^{a^{\prime}} E_{\nu}{ }^{b^{\prime}} \delta_{a^{\prime} b^{\prime}} \\
= & \omega^{2} \tau_{\mu \nu}+h_{\mu \nu}+\frac{1}{2} \tau_{\mu}{ }^{a} m_{\nu}{ }^{b} \eta_{a b}+\frac{1}{2} m_{\mu}{ }^{a} \tau_{\nu}{ }^{b} \eta_{a b} \\
& +\frac{1}{4 \omega^{2}} m_{\mu}{ }^{a} m_{\nu}{ }^{b} \eta_{a b}, \\
G^{\mu \nu}= & E^{\mu}{ }_{a} E^{\nu}{ }_{b} \eta^{a b}+E^{\mu}{ }_{a^{\prime}} E^{\nu}{ }_{b^{\prime}}{ }^{a^{\prime} b^{\prime}} \\
= & \frac{1}{\omega^{2}} \tau^{\mu \nu}+h^{\mu \nu}-\frac{1}{2 \omega^{2}}\left(\tau^{\nu}{ }_{b} m_{\rho}{ }^{b} h^{\rho \mu}+\tau^{\mu}{ }_{b} m_{\rho}{ }^{b} h^{\rho \nu}\right) \\
& -\frac{1}{2 \omega^{4}}\left(\tau^{\mu}{ }_{c} m^{c}{ }_{\rho} \tau^{\rho \nu}+\tau^{\nu}{ }_{d} m^{d}{ }_{\rho} \tau^{\rho \mu}\right) \\
& +\frac{1}{4 \omega^{4}} \tau^{\mu}{ }_{a} m_{\rho}{ }^{a}{ }^{\rho \sigma}{ }^{\rho \sigma} \tau^{\nu}{ }_{b} m_{\sigma}{ }^{b}+O\left(\omega^{-6}\right),
\end{aligned}
$$

where

$h^{\mu \nu}=e^{\mu}{ }_{a^{\prime}} e^{\nu} b^{\prime} \delta^{a^{\prime} b^{\prime}}, \quad h_{\mu \nu}=e_{\mu}{ }^{a^{\prime}} e_{\nu}^{b^{\prime}} \delta_{a^{\prime} b^{\prime}}, \quad \tau^{\mu \nu}=\tau^{\mu}{ }_{a} \tau^{\nu}{ }_{b} \eta^{a b}$.

As the next step we have to introduce an appropriate parametrization of NSNS two form. We suggested in [30] that it is natural to consider the following form of NSNS two form

$$
\begin{aligned}
B_{\mu \nu}= & \left(\omega \tau_{\mu}{ }^{a}-\frac{1}{2 \omega} m_{\mu}{ }^{a}\right)\left(\omega \tau_{\nu}{ }^{b}-\frac{1}{2 \omega} m_{\nu}{ }^{b}\right) \epsilon_{a b} \\
= & \omega^{2} \tau_{\mu}{ }^{a} \tau_{\nu}{ }^{b} \epsilon_{a b}-\frac{1}{2}\left(m_{\mu}{ }^{a} \tau_{\nu}{ }^{b}+\tau_{\mu}{ }^{a} m_{\nu}{ }^{b}\right) \epsilon_{a b} \\
& +\frac{1}{4 \omega^{2}} m_{\mu}{ }^{a} m_{\nu}{ }^{b} \epsilon_{a b},
\end{aligned}
$$

where

$$
\epsilon_{a b}=-\epsilon_{b a}, \quad \epsilon_{01}=1 .
$$

With the help of this definition we easily find

$$
\frac{1}{\omega^{2}} \tilde{\tau}_{F}^{2} B_{\mu \sigma} \partial_{\sigma} x^{\sigma} \tau^{\mu \nu} B_{\nu \rho} \partial_{\sigma} x^{\rho}=-\omega^{2} \tau_{F}^{2} \tau_{\mu \nu} \partial_{\sigma} x^{\mu} \partial_{\sigma} x^{\nu}
$$

and we see that this divergent contribution to the Hamiltonian constraint

$$
\frac{1}{\omega^{2}} \tilde{\tau}_{F}^{2} B_{\mu \sigma} \partial_{\sigma} x^{\sigma} \tau^{\mu \nu} B_{\nu \omega} \partial_{\sigma} x^{\omega}+\tilde{\tau}_{F}^{2} \omega^{2} \partial_{\sigma} x^{\mu} \tau_{\mu \nu} \partial_{\sigma} x^{\nu}
$$

vanishes. Then we obtain that the Hamiltonian constraint has the form in the limit $\omega \rightarrow \infty$

$$
\begin{aligned}
\mathcal{H}_{\tau}= & p_{\mu} h^{\mu \nu} p_{\nu}-2 \tau_{F} p_{\mu} \tau^{\mu}{ }_{a} \eta^{a b} \epsilon_{b c} \tau_{\rho}{ }^{c} \partial_{\sigma} x^{\rho} \\
& +2 \tau_{F} p_{\mu} h^{\mu \rho} m_{\rho}{ }^{b} \epsilon_{b d} \tau_{\rho}{ }^{d} \partial_{\sigma} x^{\rho} \\
& +2 \tau_{F}^{2} \partial_{\sigma} x^{\mu} \tau_{\mu}{ }^{c} \epsilon_{c d} \tau^{\nu}{ }_{e} \eta^{e d} m_{\nu}{ }^{a} \tau_{\rho}{ }^{b} \epsilon_{a b} \partial_{\sigma} x^{\rho} \\
& +2 \tau_{F}^{2} \partial_{\sigma} x^{\mu} \tau_{\mu}{ }^{a} \eta_{a b} m_{\nu}{ }^{b} \partial_{\sigma} x^{\nu} \\
& -\tau_{F}^{2} \partial_{\sigma} x^{\sigma} \tau_{\sigma}{ }^{b} \epsilon_{b a} m_{\mu}{ }^{a} h^{\mu \nu} m_{\nu}{ }^{c} \epsilon_{c d} \tau_{\rho}{ }^{d} \partial_{\sigma} x^{\rho} \\
& +\tau_{F}^{2} \partial_{\sigma} x^{\mu} h_{\mu \nu} \partial_{\sigma} x^{\nu} \\
\equiv & p_{\mu} h^{\mu \nu} p_{\nu}+p_{\mu} V^{\mu}+\tau_{F}^{2} \partial_{\sigma} x^{\mu} \bar{H}_{\mu \nu} \partial_{\sigma} x^{\nu} \\
V^{\mu}= & V^{\mu}{ }_{\nu} \partial_{\sigma} x^{\nu}
\end{aligned}
$$

where we identify $\tilde{\tau}_{F}$ with $\tau_{F}$ since as follows from the analysis above it is not necessary to rescale $\tau_{F}$ in order to have finite Hamiltonian in the limit $\omega \rightarrow \infty$.

We see that this form of the Hamiltonian constraint is rather complicated. For that reason it is necessary to check whether it defines consistent theory. Especially we would like to see whether Hamiltonian and spatial diffeomorphism constraints are the first class constraints. To do this we calculate Poisson algebra of constraints. As usually we introduce smeared form of these constraints

$\mathbf{T}_{\tau}(N)=\int d \sigma N \mathcal{H}_{\tau}, \quad \mathbf{T}_{\sigma}\left(N^{\sigma}\right)=\int d \sigma N^{\sigma} \mathcal{H}_{\sigma}$

and we easily find

$$
\begin{aligned}
\left\{\mathbf{T}_{\sigma}\left(N^{\sigma}\right), \mathbf{T}_{\sigma}\left(M^{\sigma}\right)\right\} & =\int d \sigma\left(N^{\sigma} \partial_{\sigma} M^{\sigma}-N^{\sigma} \partial_{\sigma} M^{\sigma}\right) p_{\mu} \partial_{\sigma} x^{\mu} \\
& =\mathbf{T}_{\sigma}\left(N^{\sigma} \partial_{\sigma} M^{\sigma}-N^{\sigma} \partial_{\sigma} M^{\sigma}\right) .
\end{aligned}
$$

In case of the calculation of the Poisson brackets of two Hamiltonian constraints the situation is more involved since the explicit calculation gives

$$
\begin{aligned}
& \left\{\mathbf{T}_{\tau}(N), \mathbf{T}_{\tau}(M)\right\} \\
& =\int d \sigma\left(N \partial_{\sigma} M-M \partial_{\sigma} N\right) \\
& \quad \times 2 \tau_{F}^{2}\left(p_{\mu} h^{\mu \nu} \bar{H}_{\nu \rho} \partial_{\sigma} x^{\rho}+\partial_{\sigma} x^{\rho} \bar{H}_{\rho \mu} h^{\mu \nu} p_{\nu}\right) \\
& \quad-2 \int d \sigma \tau_{F}\left(N \partial_{\sigma} M-M \partial_{\sigma} N\right) p_{\mu} V_{\nu}^{\mu} h^{\nu \omega} p_{\omega} \\
& \quad+\int d \sigma\left(N \partial_{\sigma} M-M \partial_{\sigma} N\right) p_{\rho} V_{\sigma}{ }_{\sigma} V_{\omega}^{\sigma} \partial_{\sigma} x^{\omega} \\
& \quad-\tau_{F}^{2} \int d \sigma\left(N \partial_{\sigma} M-M \partial_{\sigma} N\right)\left(V_{\nu}^{\mu} \partial_{\sigma} x^{\nu} \bar{H}_{\mu \rho} \partial_{\sigma} x^{\rho}\right. \\
& \left.\quad+\partial_{\sigma} x^{\rho} \bar{H}_{\rho \mu} V^{\mu}{ }_{\nu} \partial_{\sigma} x^{\nu}\right) .
\end{aligned}
$$


To proceed further we calculate

$$
\begin{aligned}
& 2 p_{\mu} h^{\mu \nu} \bar{H}_{\nu \rho} \partial_{\sigma} x^{\rho}+2 \partial_{\sigma} x^{\rho} \bar{H}_{\rho \mu} h^{\mu \nu} p_{\nu} \\
& =4 \tau_{F}^{2} p_{\mu} h^{\mu \nu} h_{\nu \rho} \partial_{\sigma} x^{\rho}+4 \tau_{F}^{2} \partial_{\sigma} x^{\mu} \tau_{\mu}{ }^{a} \eta_{a b} m_{\nu}{ }^{b} h^{\nu \rho} p_{\rho}, \\
& p_{\rho} V^{\rho}{ }_{\mu} V^{\mu}{ }_{\nu} \partial_{\sigma} x^{\nu}=4 \tau_{F}^{2} p_{\mu} \tau^{\mu \nu} \tau_{\nu \rho} \partial_{\sigma} x^{\rho} \\
& \quad-4 \tau_{F}^{2} p_{\mu} h^{\mu \nu} m_{\nu}{ }^{a} \tau_{\rho}{ }^{b} \eta_{a b} \partial_{\sigma} x^{\rho}, \\
& V^{\mu}{ }_{\nu} \partial_{\sigma} x^{\nu} \bar{H}_{\mu \rho} \partial_{\sigma} x^{\rho}+\partial_{\sigma} x^{\rho} \bar{H}_{\rho \mu} V^{\mu}{ }_{\nu} \partial_{\sigma} x^{\nu}=0, \\
& p_{\mu} V^{\mu}{ }_{\nu} h^{\nu \omega} p_{\omega}=0 .
\end{aligned}
$$

Collecting these results together we finally obtain

$$
\left\{\mathbf{T}_{\tau}(N), \mathbf{T}_{\tau}(M)\right\}=\mathbf{T}_{\sigma}\left(\left(N \partial_{\sigma} M-M \partial_{\sigma} N\right) 4 \tau_{F}^{2}\right)
$$

which is the correct form of the Poisson bracket between Hamiltonian constraints. Finally we calculate the Poisson bracket

$$
\left\{\mathbf{T}_{\sigma}\left(N^{\sigma}\right), \mathbf{T}_{\tau}(M)\right\}
$$

Since

$$
\begin{aligned}
\left\{\mathbf{T}_{\sigma}\left(N^{\sigma}\right), x^{\mu}\right\} & =-N^{\sigma} \partial_{\sigma} x^{\mu}, \\
\left\{\mathbf{T}_{\sigma}\left(N^{\sigma}\right), p_{\mu}\right\} & =-\partial_{\sigma} N^{\sigma} p_{\mu}-N^{\sigma} \partial_{\sigma} p_{\mu}
\end{aligned}
$$

we easily find

$$
\left\{\mathbf{T}_{\sigma}\left(N^{\sigma}\right), \mathcal{H}_{\tau}\right\}=-2 \partial_{\sigma} N^{\sigma} \mathcal{H}_{\tau}-N^{\sigma} \partial_{\sigma} \mathcal{H}_{\tau}
$$

or alternatively

$$
\left\{\mathbf{T}_{\sigma}\left(N^{\sigma}\right), \mathbf{T}_{\tau}(M)\right\}=\mathbf{T}_{\sigma}\left(N^{\sigma} \partial_{\sigma} M-\partial_{\sigma} N^{\sigma} M\right) .
$$

We see that all Poisson brackets (18), (21), and (25) vanish on the constraint surface $\mathcal{H}_{\tau} \approx 0, \mathcal{H}_{\sigma} \approx 0$ and hence they are the first class constraints and the nonrelativistic string is a well-defined system from the canonical point of view.

\section{LAGRANGIAN FORM}

In this section we focus on the Lagrangian formulation of the proposed Hamiltonian form of nonrelativistic string in Newton-Cartan background. Recall that this string is defined with the Hamiltonian constraint (16) and the spatial diffeomorphism constraint $\mathcal{H}_{\sigma} \approx 0$. In order to understand subtle points in the transformation from the Hamiltonian to Lagrangian description of this system we first start with the simpler problem of nonrelativistic string in the flat background.

\section{A. Flat space-time limit}

The nonrelativistic string in the flat background has following Hamiltonian

$$
H=\int d \sigma\left(\lambda^{\tau} \mathcal{H}_{\tau}+\lambda^{\sigma} \mathcal{H}_{\sigma}\right)
$$

where

$\mathcal{H}_{\tau}=-2 \tau_{F} p_{a} \eta^{a b} e_{b c} \partial_{\sigma} x^{c}+p_{i} h^{i j} p_{j}+\tau_{F}^{2} h_{i j} \partial_{\sigma} x^{i} \partial_{\sigma} x^{j}$,

$\mathcal{H}_{\sigma}=p_{i} \partial_{\sigma} x^{i}+p_{a} \partial_{\sigma} x^{a}$,

where $a, b, c, \ldots=0,1$ and where $h_{i j}=\delta_{i j}, h^{i j}=\delta^{i j}$, $i, j, \ldots=2, \ldots, d-1$. Our goal is to find a Lagrangian formulation of the nonrelativistic string in flat background. With the help of the Hamiltonian (26) we obtain the following equations of motion for $x^{0}, x^{1}$ and $x^{i}$

$$
\begin{aligned}
& \partial_{\tau} x^{0}=\left\{x^{0}, H\right\}=-2 \tau_{F} \lambda^{\tau} \partial_{\sigma} x^{1}+\lambda^{\sigma} \partial_{\sigma} x^{0}, \\
& \partial_{\tau} x^{1}=\left\{x^{1}, H\right\}=-2 \tau_{F} \lambda^{\tau} \partial_{\sigma} x^{0}+\lambda^{\sigma} \partial_{\sigma} x^{1}, \\
& \partial_{\tau} x^{i}=\left\{x^{i}, H\right\}=2 \lambda^{\tau} h^{i j} p_{j}+\lambda^{\sigma} \partial_{\sigma} x^{i} .
\end{aligned}
$$

Then it is easy to find the corresponding Lagrangian density

$$
\begin{aligned}
\mathcal{L}= & p_{a} \partial_{\tau} x^{a}+p_{i} \partial_{\tau} x^{i}-\mathcal{L}=\lambda^{\tau} p_{i} h^{i j} p_{j}-\lambda^{\tau} \partial_{\sigma} x^{i} \partial_{\sigma} x^{j} h_{i j} \\
= & \frac{1}{4 \lambda^{\tau}}\left(\partial_{\tau} x^{i}-\lambda^{\sigma} \partial_{\sigma} x^{i}\right) h_{i j}\left(\partial_{\tau} x^{j}-\lambda^{\sigma} \partial_{\sigma} x^{j}\right) \\
& -\lambda^{\tau} \tau_{F}^{2} h_{i j} \partial_{\sigma} x^{i} \partial_{\sigma} x^{j}
\end{aligned}
$$

We see that this Lagrangian does not depend on the variables $x^{a}$ which is confusing since if we perform inverse Legendre transformation from (29) and determine the corresponding Hamiltonian we will find that it does not depend on $p_{a}$. We can resolve this problem when we closely examine equations of motion for $x^{0}$ and $x^{1}$. We first consider the first equation in (28) and multiply it with $\partial_{\sigma} x^{0}$ while we multiply the second one with $\partial_{\sigma} x^{1}$. Then if we take their difference we obtain

$-\partial_{\tau} x^{0} \partial_{\sigma} x^{0}+\partial_{\tau} x^{1} \partial_{\sigma} x^{1}=\lambda^{\sigma}\left(\partial_{\sigma} x^{1} \partial_{\sigma} x^{1}-\partial_{\sigma} x^{0} \partial_{\sigma} x^{0}\right)$

that can be solved for $\lambda^{\sigma}$ as

$$
\lambda^{\sigma}=\frac{\mathbf{a}_{\tau \sigma}}{\mathbf{a}_{\sigma \sigma}}, \quad \mathbf{a}_{\alpha \beta}=\partial_{\alpha} x^{a} \partial_{\beta} x^{b} \eta_{a b} .
$$

On the other hand, from the equations of motion for $x^{0}$ and $x^{1}$ we obtain

$$
\begin{aligned}
& \left(\partial_{\tau} x^{0}-\lambda^{\sigma} \partial_{\sigma} x^{0}\right)^{2}=4\left(\lambda^{\tau}\right)^{2} \partial_{\sigma} x^{1} \partial_{\sigma} x^{1}, \\
& \left(\partial_{\tau} x^{1}-\lambda^{\sigma} \partial_{\sigma} x^{1}\right)^{2}=4\left(\lambda^{\tau}\right)^{2} \partial_{\sigma} x^{0} \partial_{\sigma} x^{0}
\end{aligned}
$$

that implies

$$
-\mathbf{a}_{\tau \tau}+2 \lambda^{\sigma} \mathbf{a}_{\sigma \tau}-\left(\lambda^{\sigma}\right)^{2} \mathbf{a}_{\sigma \sigma}=4\left(\lambda^{\tau}\right)^{2} \mathbf{a}_{\sigma \sigma} \tau_{F}^{2} .
$$


Inserting (31) into this equation we find that $\lambda^{\tau}$ is equal to

$$
\lambda^{\tau}=\frac{\sqrt{-\operatorname{det} \mathbf{a}_{\alpha \beta}}}{2 \tau_{F} \mathbf{a}_{\sigma \sigma}} .
$$

Then if we combine (31) together with (33) we get

$$
\begin{aligned}
& \frac{1}{\lambda^{\tau}}=-2 \tau_{F} \mathbf{a}^{\tau \tau} \sqrt{-\operatorname{det} \mathbf{a}}, \quad \frac{2 \lambda^{\sigma}}{\lambda^{\tau}}=4 \tau_{F} \mathbf{a}^{\tau \sigma} \sqrt{-\operatorname{det} \mathbf{a}}, \\
& \frac{\left(\lambda^{\sigma}\right)^{2}}{4\left(\lambda^{\tau}\right)^{2}}-\lambda^{\tau} \tau_{F}^{2}=-\tau_{F} \mathbf{a}^{\sigma \sigma} \sqrt{-\operatorname{det} \mathbf{a}} .
\end{aligned}
$$

Finally inserting (34) into (29) we obtain

$$
\mathcal{L}=-\frac{\tau_{F}}{2} \sqrt{-\operatorname{det} \mathbf{a}} \mathbf{a}^{\alpha \beta} h_{\alpha \beta}
$$

which has exactly the same form as the Lagrangian density that was derived in [26].

\section{B. Lagrangian for string in Newton-Cartan background}

Now we proceed to the case of the nonrelativistic string in Newton-Cartan background. As the first step we formulate the Hamiltonian constraint with the help of the variables that reflect an invariance of the theory under generalized Galilean boosts that have the form [26]

$$
\delta e_{\mu}{ }^{a^{\prime}}=\tau_{\mu}{ }^{a} \lambda_{a}{ }^{a^{\prime}}, \quad \delta \tau^{\mu}{ }_{a}=e_{a^{\prime}} \lambda^{a^{\prime}}{ }_{a}, \quad \delta m_{\mu}{ }^{a}=e_{\mu}{ }^{a^{\prime}} \lambda_{a^{\prime}}{ }^{a},
$$

where $\lambda_{a}{ }^{a^{\prime}}$ are parameters that obey the following relations

$$
\begin{aligned}
\eta_{a c} \lambda^{c}{ }_{a^{\prime}}+\delta_{a^{\prime} b^{\prime}} \lambda^{b^{\prime}} & =0, & & \lambda_{a^{\prime}}{ }^{c} \eta_{c a}+\lambda_{a}{ }^{b^{\prime}} \delta_{b^{\prime} a^{\prime}}=0, \\
\lambda_{a^{\prime}}{ }^{a}+\lambda^{a}{ }_{a^{\prime}} & =0, & & \lambda^{a^{\prime}}{ }_{a}+\lambda_{a}{ }^{a^{\prime}}=0 .
\end{aligned}
$$

Now we define boost invariant temporary vierbein as $\hat{\tau}^{\mu}{ }_{a}$

$$
\hat{\tau}^{\mu}{ }_{a}=\tau^{\mu}{ }_{a}-h^{\mu \nu} m_{\nu}{ }^{b} \eta_{b a} .
$$

This is invariant under (36) since

$\delta \hat{\tau}_{\mu}{ }^{a}=e_{a^{\prime}}^{\mu} \lambda^{a^{\prime}}{ }_{a}-e^{\mu}{ }_{c^{\prime}} \delta^{c^{\prime} b^{\prime}} \lambda_{b^{\prime}}{ }^{b} \eta_{b a}=e^{\mu}{ }_{a^{\prime}} \lambda^{a^{\prime}}{ }_{a}+e^{\mu}{ }_{a^{\prime}} \lambda_{a}{ }^{a^{\prime}}=0$,

where in the last step we used (37). With the help of $\hat{\tau}_{\mu}{ }^{a}$ we can rewrite $V^{\mu}$ into manifestly invariant form

$$
V^{\mu}=V^{\mu}{ }_{\nu} \partial_{\sigma} x^{\nu}, \quad V_{\nu}^{\mu}=-2 \tau_{F} \hat{\tau}^{\mu}{ }_{a} \epsilon^{a b} \hat{\tau}^{\sigma}{ }_{b} \tau_{\sigma \nu}=V^{\mu \sigma} \tau_{\sigma \nu}
$$

where $\epsilon^{a b} \equiv \eta^{a c} \eta^{b d} \epsilon_{c d}$ and where $V^{\mu \nu}=-V^{\nu \mu}$. Let us now analyze in more detail the object $\bar{H}_{\mu \nu}$. After some calculations we obtain that it can be rewritten into the form

$$
\begin{aligned}
\bar{H}_{\mu \nu} & =-\tau_{F}^{2} \tau_{\mu}{ }^{c} \epsilon_{c d} \Phi^{d a} \epsilon_{a b} \tau_{\nu}{ }^{b}+\tau_{F}^{2} \bar{h}_{\mu \nu}, \\
\bar{h}_{\mu \nu} & =h_{\mu \nu}+m_{\mu}{ }^{a} \tau_{\nu}{ }^{b} \eta_{a b}+\tau_{\mu}{ }^{a} m_{\nu}{ }^{b} \eta_{a b}, \\
\Phi^{a b} & =-\tau^{\mu}{ }_{d} \eta^{d a} m_{\mu}{ }^{b}-m_{\mu}{ }^{a} \tau^{\mu}{ }_{d} \eta^{d b}+m_{\mu}{ }^{a} h^{\mu \nu} m_{\nu}{ }^{b} .
\end{aligned}
$$

An important property of (41) is that it is written with the help of the objects that are invariant under (36). To see this let us first consider variation of $\bar{h}_{\mu \nu}$

$$
\begin{aligned}
\delta \bar{h}_{\mu \nu}= & \tau_{\mu}{ }^{a} \lambda_{a}{ }^{a^{\prime}} \delta_{a^{\prime} b^{\prime}} e_{\nu}{ }^{b^{\prime}}+e_{\mu}{ }^{a^{\prime}} \delta_{a^{\prime} b^{\prime}} \tau_{\nu}{ }^{b} \lambda_{b}{ }^{b^{\prime}} \\
& +e_{\mu}{ }^{{ }^{\prime}} \lambda_{a^{\prime}}{ }^{a} \tau_{\nu}{ }^{b} \eta_{a b}+\tau_{\mu}{ }^{a} \eta_{a b} e_{\nu}{ }^{b^{\prime}} \lambda_{b^{\prime}}{ }^{b}=0,
\end{aligned}
$$

where we used (37). Finally we consider the variation of $\Phi^{a b}=\Phi^{b a}$. Note that the matrix $\Phi^{a b}$ can be interpreted as the matrix of Newton-potential in generalized NewtonCartan gravity which is now matrix valued as opposite to the scalar form of $\Phi$ in ordinary Newton-Cartan gravity. On the other hand it is still invariant under (36) since

$$
\begin{aligned}
& \delta \Phi^{a b}=-e^{\mu}{ }_{a^{\prime}} \lambda^{a^{\prime}}{ }_{c} m_{\mu}{ }^{b} \eta^{c a}-m_{\mu}{ }^{a} e^{\mu}{ }_{a^{\prime}} \lambda^{a^{\prime}}{ }_{d} \eta^{d b} \\
& +e^{\nu}{ }_{d^{\prime}} \delta^{c^{\prime} a^{\prime}} \lambda_{a^{\prime}}{ }^{a} m_{\nu}{ }^{b}+m_{\mu}{ }^{a} e^{\mu}{ }_{c^{\prime}} \delta^{c^{\prime} b^{\prime}} \lambda_{b^{\prime}}{ }^{b} \\
& =-e^{\mu}{ }_{a^{\prime}} \lambda^{a^{\prime}}{ }_{c} m_{\mu}{ }^{b} \eta^{c a}-m_{\mu}{ }^{a} e^{\mu}{ }_{a^{\prime}} \lambda^{a^{\prime}}{ }_{d} \eta^{d b} \\
& -e^{\nu}{ }_{c^{\prime}} \delta^{c^{\prime} a^{\prime}} \lambda^{a}{ }_{a^{\prime}} m_{\nu}{ }^{b}-m_{\mu}{ }^{a} e^{\mu}{ }_{c^{\prime}} c^{c^{\prime} b^{\prime}} \lambda^{b}{ }_{b^{\prime}} \\
& =-e^{\mu}{ }_{a^{\prime}} \lambda^{a^{\prime}}{ }_{c} m_{\mu}{ }^{b} \eta^{c a}-m_{\mu}{ }^{a} e^{\mu}{ }_{a^{\prime}} \lambda^{a^{\prime}}{ }_{d} \eta^{d b} \\
& +e^{\mu}{ }_{c^{\prime}} \lambda^{c^{\prime}}{ }_{c} \eta^{c a} m_{\mu}{ }^{b}+m_{\mu}{ }^{a} e^{\mu}{ }_{c^{\prime}} \lambda^{c^{\prime}}{ }_{c} \eta^{c b}=0,
\end{aligned}
$$

where in the first step we used the relation $\lambda_{a^{\prime}}{ }^{a}=-\lambda^{a}{ }_{a^{\prime}}$ and in the last step we used the fact that

$$
\lambda^{c^{\prime}}{ }_{b} \eta^{b a}+\lambda^{c}{ }_{b^{\prime}} \delta^{b^{\prime} c^{\prime}}=0 .
$$

It is also instructive to elaborate more about an expression that contain the potential $\Phi^{a b}$. We find that after some calculations it can be rewritten into the form

$$
\begin{aligned}
& -\tau_{F}^{2} \partial_{\sigma} x^{\mu} \tau_{\mu}{ }^{c} \epsilon_{c d} \Phi^{d a} \epsilon_{a b} \tau^{b}{ }_{\nu} \partial_{\sigma} x^{\nu} \\
& =\tau_{F}^{2} \partial_{\sigma} x^{\mu} \tau_{\mu}{ }^{c} \eta_{c a} \Phi^{a b} \eta_{b d} \tau_{\nu}{ }^{d} \partial_{\sigma} x^{\nu}-\tau_{F}^{2} \mathbf{a}_{\sigma \sigma} \Phi^{a b} \eta_{b a}
\end{aligned}
$$

so that we finally obtain manifestly invariant Hamiltonian in the form

$$
\begin{aligned}
H= & \int d \sigma\left(\lambda^{\tau} \mathcal{H}_{\tau}+\lambda^{\sigma} \mathcal{H}_{\sigma}\right), \quad \mathcal{H}_{\sigma}=p_{\mu} \partial_{\sigma} x^{\mu}, \\
\mathcal{H}_{\tau}= & p_{\mu} h^{\mu \nu} p_{\nu}-2 p_{\mu} \tau_{F} \hat{\tau}^{\mu}{ }_{a} \epsilon^{a b} \hat{\tau}^{\sigma}{ }_{b} \tau_{\sigma \nu} \partial_{\sigma} x^{\nu} \\
& +\tau_{F}^{2} \tau^{c} \eta_{c a} \Phi^{a b} \eta_{b d} \tau^{d}-\tau_{F}^{2} \mathbf{a}_{\sigma \sigma} \Phi^{a b} \eta_{b a}+\tau_{F}^{2} \bar{h}_{\sigma \sigma},
\end{aligned}
$$

where $\tau^{a}=\partial_{\sigma} x^{\mu} \tau_{\mu}{ }^{a}, \bar{h}_{\sigma \sigma}=\partial_{\sigma} x^{\mu} \bar{h}_{\mu \nu} \partial_{\sigma} x^{\nu}$ and where $\lambda^{\tau}$ and $\lambda^{\sigma}$ are corresponding Lagrange multipliers. 
Before we proceed to the Lagrangian formulation of the theory let us introduce vierbein $\hat{e}_{\mu}{ }^{a^{\prime}}$ defined as

$$
\hat{e}_{\mu}{ }^{a^{\prime}}=e_{\mu}{ }^{a^{\prime}}+m_{\nu}{ }^{a} e^{\nu}{ }_{c^{\prime}} \delta^{c^{\prime} a^{\prime}} \tau_{\mu}{ }^{b} \eta_{b a},
$$

that is again invariant under (36)

$$
\begin{aligned}
\delta \hat{e}_{\mu}{ }^{a^{\prime}} & =\tau_{\mu}{ }^{a} \lambda_{a}{ }^{a^{\prime}}+\lambda_{c^{\prime}}{ }^{a} \delta^{c^{\prime} a^{\prime}} \tau_{\mu}{ }^{b} \eta_{b a} \\
& =\tau_{\mu}{ }^{a} \lambda_{a}{ }^{a^{\prime}}-\lambda_{b}{ }^{b^{\prime}} \delta_{b^{\prime} c^{\prime}} \delta^{c^{\prime} a^{\prime}} \tau_{\mu}{ }^{b}=0 .
\end{aligned}
$$

Note that we have the following useful identity

$$
\hat{\tau}^{\mu}{ }_{a} \hat{e}_{\mu}{ }^{\prime}=0
$$

and also

$$
\hat{e}_{\mu}{ }^{a^{\prime}} e_{b^{\prime}}^{\mu}=\delta^{a^{\prime}}{ }_{b^{\prime}}, \quad \hat{e}_{\mu}{ }^{a^{\prime}} h^{\mu \nu}=e^{\nu}{ }_{c^{\prime}} \delta^{c^{\prime} a^{\prime}} .
$$

Now we are ready to proceed to the Lagrangian formulation of the theory. We begin with the canonical equations of motion for $x^{\mu}$ that follow from the Hamiltonian (46)

$$
\partial_{\tau} x^{\mu}=\lambda^{\tau}\left(2 h^{\mu \nu} p_{\nu}+V^{\mu}\right)+\lambda^{\sigma} \partial_{\sigma} x^{\nu} .
$$

Let us now multiply this equation with $\hat{e}_{\mu}{ }^{a^{\prime}}$. Using the fact that $\hat{e}_{\mu}{ }^{a^{\prime}} \hat{\tau}^{\mu}{ }_{b^{\prime}}=0$ we find that $\hat{e}_{\mu}{ }^{a^{\prime}} V^{\mu}=0$ and from (51) we obtain

$$
\hat{e}_{\mu}{ }^{a^{\prime}} \partial_{\tau} x^{\mu}=2 \lambda^{\tau} e^{\mu}{ }_{c^{\prime}} c^{c^{\prime} a^{\prime}} p_{\mu}+\lambda^{\sigma} \hat{e}_{\mu}{ }^{a^{\prime}} \partial_{\sigma} x^{\mu}
$$

and consequently

$$
\begin{aligned}
& \left(\partial_{\tau} x^{\mu}-\lambda^{\sigma} \partial_{\sigma} x^{\mu}\right) \hat{e}_{\mu}{ }^{\prime} \delta_{a^{\prime} b^{\prime}} \hat{e}_{\nu} b^{\prime}\left(\partial_{\tau} x^{\nu}-\lambda^{\sigma} \partial_{\sigma} x^{\nu}\right) \\
& \quad=4\left(\lambda^{\tau}\right)^{2} p_{\mu} h^{\mu \nu} p_{\nu} .
\end{aligned}
$$

With the help of this result we easily find the Lagrangian density in the form

$$
\begin{aligned}
\mathcal{L}= & p_{\mu} \partial_{\tau} x^{\mu}-\lambda^{\tau} \mathcal{H}_{\tau}-\lambda^{\sigma} \mathcal{H}_{\sigma} \\
= & \frac{1}{4 \lambda^{\tau}}\left(\partial_{\tau} x^{\mu}-\lambda^{\sigma} \partial_{\sigma} x^{\mu}\right) \hat{e}_{\mu}{ }^{a^{\prime}} \delta_{a^{\prime} b^{\prime}} \hat{e}_{\nu}{ }^{b^{\prime}}\left(\partial_{\tau} x^{\nu}-\lambda^{\sigma} \partial_{\sigma} x^{\nu}\right) \\
& -\tau_{F}^{2} \lambda^{\tau} \bar{H}_{\sigma \sigma} .
\end{aligned}
$$

To proceed further we observe that (51) implies

$$
\hat{e}_{\mu}{ }^{a^{\prime}} \delta_{a^{\prime} b^{\prime}} \hat{e}_{\nu}^{b^{\prime}}=\bar{h}_{\mu \nu}+\tau_{\mu}{ }^{c} \eta_{c a} \Phi^{a b} \eta_{b d} \tau_{\nu}{ }^{d},
$$

where we also used

$$
e_{\mu}{ }^{\prime} e^{\nu}{ }_{a^{\prime}}=\delta_{\mu}^{\nu}-\tau_{\mu}{ }^{a} \tau_{a}^{\nu} .
$$

Then we can rewrite the Lagrangian density (54) into the form

$$
\begin{aligned}
\mathcal{L}= & \frac{1}{4 \lambda^{\tau}}\left(\bar{h}_{\tau \tau}-2 \lambda^{\sigma} \bar{h}_{\sigma \tau}+\left(\lambda^{\sigma}\right)^{2} \bar{h}_{\sigma \sigma}\right. \\
& +\partial_{\tau} x^{\mu} \tau_{\mu}{ }^{c} \eta_{c a} \Phi^{a b} \eta_{b d} \tau_{\nu}{ }^{d} \partial_{\tau} x^{\nu} \\
& -2 \lambda^{\sigma} \partial_{\tau} x^{\mu} \tau_{\mu}{ }^{c} \eta_{c a} \Phi^{a b} \eta_{b d} \tau_{\nu}{ }^{d} \partial_{\sigma} x^{\nu} \\
& \left.+\left(\lambda^{\sigma}\right)^{2} \partial_{\sigma} x^{\mu} \tau_{\mu}{ }^{c} \eta_{c a} \Phi^{a b} \eta_{b d} \tau_{\nu}{ }^{d} \partial_{\sigma} x^{\nu}\right) \\
& -\lambda^{\tau} \tau_{F}^{2} \partial_{\sigma} x^{\mu} \tau_{\mu}{ }^{c} \eta_{c a} \Phi^{a b} \eta_{b d} \tau_{\nu}{ }^{d} \partial_{\sigma} x^{\nu} \\
& +\lambda^{\tau} \tau_{F}^{2} \mathbf{a}_{\sigma \sigma} \Phi^{a b} \eta_{b a}-\lambda^{\tau} \tau_{F}^{2} \bar{h}_{\sigma \sigma},
\end{aligned}
$$

where $\bar{h}_{\alpha \beta}=\bar{h}_{\mu \nu} \partial_{\alpha} x^{\mu} \partial_{\beta} x^{\nu}$.

Finally we eliminate $\lambda^{\tau}$ and $\lambda^{\sigma}$ from (57). As in the case of the flat space-time limit, their form is not determined by their equations of motion. Instead they can be determined using the equations of motion for $x^{\mu}$. In fact, if we multiply (51) by $\tau_{\mu \nu}$ and use the fact that $\tau_{\mu \nu} h^{\nu \rho}=0$ we obtain

$$
\tau_{\mu \nu}\left(\partial_{\tau} x^{\nu}-\lambda^{\sigma} \partial_{\sigma} x^{\nu}\right)-\lambda^{\tau} \tau_{\mu \nu} V^{\nu}=0 .
$$

We can multiply this equation with $\partial_{\sigma} x^{\mu}$ and we obtain

$$
\lambda^{\sigma}=\frac{\mathbf{a}_{\sigma \tau}}{\mathbf{a}_{\sigma \sigma}}, \quad \mathbf{a}_{\alpha \beta}=\partial_{\alpha} x^{\mu} \tau_{\mu \nu} \partial_{\beta} x^{\nu}
$$

using the fact that

$$
\partial_{\sigma} x^{\mu} \tau_{\mu \nu} V^{\nu}=2 \tau_{F} \partial_{\sigma} x^{\mu} \tau_{\mu}{ }^{a} \epsilon_{a b} \tau_{\nu}{ }^{b} \partial_{\sigma} x^{\nu}=0 .
$$

In a similar way we obtain

$$
\left(\partial_{\tau} x^{\mu}-\lambda^{\sigma} \partial_{\sigma} x^{\mu}\right) \tau_{\mu \nu}\left(\partial_{\tau} x^{\nu}-\lambda^{\sigma} \partial_{\sigma} x^{\nu}\right)=\left(\lambda^{\tau}\right)^{2} V^{\mu} \tau_{\mu \nu} V^{\nu}
$$

that can be solved for $\lambda^{\tau}$ as

$$
\lambda^{\tau}=\frac{\sqrt{-\operatorname{det} \mathbf{a}_{\alpha \beta}}}{\sqrt{-V^{\mu} \tau_{\mu \nu} V^{\nu}} \sqrt{\mathbf{a}_{\sigma \sigma}}},
$$

where

$$
V^{\mu} \tau_{\mu \nu} V^{\nu}=-4 \tau_{F}^{2} \mathbf{a}_{\sigma \sigma} .
$$

Now we see that we can proceed as in the case of the nonrelativistic string in flat space-time and we obtain the final result

$$
\begin{aligned}
\mathcal{L}= & -\frac{\tau_{F}}{2} \sqrt{-\operatorname{det} \mathbf{a}} \\
& \times\left(\mathbf{a}^{\alpha \beta} \bar{h}_{\alpha \beta}+\mathbf{a}^{\alpha \beta} \partial_{\alpha} x^{\mu} \tau_{\mu}{ }^{c} \eta_{c a} \Phi^{a b} \eta_{b d} \tau_{\nu}{ }^{d} \partial_{\beta} x^{\nu}-\Phi^{a b} \eta_{b a}\right)
\end{aligned}
$$

We see that this Lagrangian density almost coincides with the Lagrangian density found [26] up to terms that contain matrix valued Newton potential $\Phi_{a b}$. Now we are going to argue that these terms cancel each other. In fact, note that $\mathbf{a}_{\alpha \beta}$ is defined as

$$
\mathbf{a}_{\alpha \beta}=\tau_{\alpha}{ }^{a} \tau_{\beta}{ }^{b} \eta_{a b}
$$


where $\tau_{\alpha}{ }^{a} \equiv \partial_{\alpha} x^{\mu} \tau_{\mu}{ }^{a}$ is $2 \times 2$ matrix. Since $\tau_{\alpha \beta}$ is nonsingular so that $\tau_{\alpha}{ }^{a}$ is nonsingular as well due to the fact that

$$
\operatorname{det} \mathbf{a}_{\alpha \beta}=\left(\operatorname{det} \tau_{\alpha}{ }^{a}\right)^{2} \operatorname{det} \eta_{a b}=-\left(\operatorname{det} \tau_{\alpha}{ }^{a}\right)^{2} \neq 0 .
$$

Then we can introduce an inverse matrix $\tau_{a}^{\beta}$ that obeys the relation

$$
\tau^{\alpha}{ }_{a} \tau_{\alpha}{ }^{b}=\delta_{a}{ }^{b} .
$$

As a result we can define $\mathbf{a}^{\alpha \beta}$ as

$$
\mathbf{a}^{\alpha \beta}=\tau_{a}^{\alpha} \tau_{b}^{\beta} \eta^{a b}
$$

that obeys

$$
\mathbf{a}^{\alpha \beta} \tau_{\beta}{ }^{a}=\tau^{\alpha}{ }_{c} \eta^{c a}
$$

and hence

$$
\mathbf{a}^{\alpha \beta} \tau_{\beta}{ }^{b} \tau_{\alpha}{ }^{a}=\tau_{\beta}{ }^{b} \tau^{\beta}{ }_{c} \eta^{c a}=\eta^{b a} .
$$

With the help of these results we can manipulate with the second term in (63) as

$-\frac{\tau_{F}}{2} \sqrt{-\operatorname{det} \mathbf{a}} \mathbf{a}^{\alpha \beta} \tau_{\alpha}{ }^{a} \Phi_{a b} \tau_{\beta}{ }^{b}=-\frac{\tau_{F}}{2} \sqrt{-\operatorname{det} \mathbf{a}} \eta^{a b} \Phi_{b a}$

and we see that it exactly cancels the last term in (63). As a result we derive the Lagrangian density in the final form

$$
\mathcal{L}=-\frac{T}{2} \sqrt{-\operatorname{det} \mathbf{a}} \mathbf{a}^{\alpha \beta} \bar{h}_{\alpha \beta}
$$

which is Lagrangian density proposed in [26]. This result again confirms validity of our approach.

\section{CONCLUSION}

Let us outline our results and suggest possible extension of this work. We found a Hamiltonian for nonrelativistic string in Newton-Cartan background from the Hamiltonian of relativistic string in general background when we used the limiting procedure introduced in [15]. The corresponding Hamiltonian is a linear combination of two constraints, and we checked that they are the first class constraints which is a consequence of diffeomorphism invariance of world-sheet theory. We also introduced variables that are invariant under Milne boost and we showed that the Hamiltonian constraint is invariant under this transformation too. Finally we found Lagrangian formulation of the nonrelativistic string in Newton-Cartan background that agrees with the Lagrangian density proposed in [26]. We mean that this is a very nice consistency check of our result.

This paper can be extended in different directions. It would be possible to perform similar analysis in the case of nonrelativistic p-brane in Newton-Cartan background. Second, we could also extend this analysis to the case of superstring. We hope to return to some of these problems in future.

\section{ACKNOWLEDGMENTS}

This work was supported by the Grant Agency of the Czech Republic under the Grant No. P201/12/G028.
[1] M. H. Christensen, J. Hartong, N. A. Obers, and B. Rollier, Torsional Newton-Cartan geometry and Lifshitz holography, Phys. Rev. D 89, 061901 (2014).

[2] M. H. Christensen, J. Hartong, N. A. Obers, and B. Rollier, Boundary stress-energy tensor and Newton-Cartan geometry in Lifshitz holography, J. High Energy Phys. 01 (2014) 057.

[3] J. Hartong, E. Kiritsis, and N. A. Obers, Lifshitz spacetimes for Schrdinger holography, Phys. Lett. B 746, 318 (2015).

[4] S. A. Hartnoll, A. Lucas, and S. Sachdev, Holographic quantum matter, arXiv:1612.07324.

[5] D. T. Son, Toward an AdS/cold atoms correspondence: A Geometric realization of the Schrodinger symmetry, Phys. Rev. D 78, 046003 (2008).

[6] K. Balasubramanian and J. McGreevy, Gravity Duals for Non-relativistic CFTs, Phys. Rev. Lett. 101, 061601 (2008).

[7] C. P. Herzog, M. Rangamani, and S. F. Ross, Heating up Galilean holography, J. High Energy Phys. 11 (2008) 080.

[8] D. T. Son, Newton-Cartan geometry and the quantum Hall effect, arXiv:1306.0638.
[9] S. Janiszewski and A. Karch, Non-relativistic holography from Horava gravity, J. High Energy Phys. 02 (2013) 123.

[10] E. Cartan, Sur les varits connexion affine et la thorie de la relativit gnralise. (premire partie), Annales Sci. Ecole Norm. Sup. 40, 325 (1923).

[11] E. Bergshoeff, A. Chatzistavrakidis, L. Romano, and J. Rosseel, Newton-Cartan gravity and torsion, J. High Energy Phys. 10 (2017) 194.

[12] E. A. Bergshoeff and J. Rosseel, Three-Dimensional Extended Bargmann Supergravity, Phys. Rev. Lett. 116, 251601 (2016).

[13] H. R. Afshar, E. A. Bergshoeff, A. Mehra, P. Parekh, and B. Rollier, A Schrdinger approach to Newton-Cartan and HoavaLifshitz gravities, J. High Energy Phys. 04 (2016) 145.

[14] E. Bergshoeff, J. Rosseel, and T. Zojer, Newton-Cartan supergravity with torsion and Schrdinger supergravity, J. High Energy Phys. 11 (2015) 180.

[15] E. Bergshoeff, J. Rosseel, and T. Zojer, Newton-Cartan (super)gravity as a non-relativistic limit, Classical Quantum Gravity 32, 205003 (2015). 
[16] E. A. Bergshoeff, J. Hartong, and J. Rosseel, Torsional Newton-Cartan geometry and the Schrdinger algebra, Classical Quantum Gravity 32, 135017 (2015).

[17] R. Andringa, E. Bergshoeff, S. Panda, and M. de Roo, Newtonian gravity and the Bargmann algebra, Classical Quantum Gravity 28, 105011 (2011).

[18] J. Hartong and N. A. Obers, Hoava-Lifshitz gravity from dynamical Newton-Cartan geometry, J. High Energy Phys. 07 (2015) 155.

[19] J. Hartong, Y. Lei, and N. A. Obers, Nonrelativistic ChernSimons theories and three-dimensional Hoava-Lifshitz gravity, Phys. Rev. D 94, 065027 (2016).

[20] E. Bergshoeff, J. Gomis, B. Rollier, J. Rosseel, and T. ter Veldhuis, Carroll versus Galilei gravity, J. High Energy Phys. 03 (2017) 165.

[21] K. T. Grosvenor, J. Hartong, C. Keeler, and N. A. Obers, Homogeneous nonrelativistic geometries as coset spaces, Classical Quantum Gravity 35, 175007 (2018).

[22] K. Jensen, On the coupling of Galilean-invariant field theories to curved spacetime, SciPost Phys. 5, 011 (2018).
[23] K. Jensen, Aspects of hot Galilean field theory, J. High Energy Phys. 04 (2015) 123.

[24] K. Jensen and A. Karch, Revisiting non-relativistic limits, J. High Energy Phys. 04 (2015) 155.

[25] P. Horava, Quantum gravity at a Lifshitz point, Phys. Rev. D 79, 084008 (2009).

[26] R. Andringa, E. Bergshoeff, J. Gomis, and M. de Roo, 'Stringy' Newton-Cartan gravity, Classical Quantum Gravity 29, 235020 (2012).

[27] T. Harmark, J. Hartong, and N. A. Obers, Nonrelativistic strings and limits of the AdS/CFT correspondence, Phys. Rev. D 96, 086019 (2017).

[28] A. Barducci, R. Casalbuoni, and J. Gomis, Non-relativistic spinning particle in a Newton-Cartan background, J. High Energy Phys. 01 (2018) 002.

[29] J. Kluson, Canonical analysis of non-relativistic particle and superparticle, Eur. Phys. J. C 78, 117 (2018).

[30] J. Kluson, Note about Hamiltonian formalism for Newton-Cartan string and p-brane, Eur. Phys. J. C 78, 511 (2018). 